\title{
DOI: https://doi.org/10.24297/jam.v19i.8876
}

\section{A new modified homotopy perturbation method for fractional partial differential equations with proportional delay}

\author{
Ahmad. A. H. Mtawal ${ }^{1}$, Sameehah. R. Alkaleeli² \\ 1Department of Mathematics, Faculty of Education Almarj, Benghazi University, Almarj, Libya \\ 2Department of Mathematics, Faculty of Arts and Science Almarj, Benghazi University, Almarj, Libya \\ Hussanahmad65@yahoo.com¹ and sameehah.alkaleeli@uob.edu.ly²
}

\begin{abstract}
In this paper, we suggest and analyze a technique by combining the Shehu transform method and the homotopy perturbation method. This method is called the Shehu transform homotopy method (STHM). This method is used to solve the time-fractional partial differential equations (TFPDEs) with proportional delay. The fractional derivative is described in Caputo's sense. The solutions proposed in the series converge rapidly to the exact solution. Some examples are solved to show the STHM is easy to apply.
\end{abstract}

Keywords: Shehu transforms method, Homotopy perturbation method, time-fractional partial differential equations TFPDEs with proportional delay.

\section{Introduction}

Due to its broad variety of applications in various practical fields such as fluid dynamics, signal processing, electrical grids, diffusion, reaction processes and others in science and engineering $[5,12,18]$, fractional differential equation has become very important among researchers. Of these, nonlinear earthquake oscillations may be modeled on fractional derivatives [7], with fractional derivatives in the fluid-dynamic traffic model [8]. Indeed, an exact solution of a broad class of the differential equation is too difficult to find. Different types of vigorous techniques have been developed recent years to find an approximate solution to this type of fractional model differential equations, such as general differential transform method [13], Variational iteration method [21, 24], Adomian decomposition method [20], Homotopy perturbation method [19, 25], Homotopy perturbation Sumudu transform method [14, 28], Homotopy analysis method [23], Local fractional variational iteration method [37], Variaitional homotopy perturbation method [15] and Fractional reduced differential transform method [26, 31, 32, 33]. In the recent, vigourous techniques eith Shehu transform has been developed, among them, see $[11,34,39]$. The partial functional differential equations with proportional delays, a special class of delay partial differential equation, arise specially in the field of biology medicine, population ecology, control systems and climate methods [36], and complex economic macrodynamics [10]. In this paper, we get the numerical solution of the initial valued autonomous system of TFPDEs with proportional delay $[25,29,30]$ defined by

$$
\begin{gathered}
D_{t}^{\alpha}(\psi(x, t))=f\left(x, t, \psi\left(a_{0} x, b_{0} t\right), \frac{\partial}{\partial x} \psi\left(a_{1} x, b_{1} t\right), \ldots, \frac{\partial^{m}}{\partial x^{m}} \psi\left(a_{m} x, b_{m} t\right)\right), \\
\psi^{k}(x, 0)=\varphi_{k}(x) .
\end{gathered}
$$

Where $a_{i}, b_{i} \in(0,1)$ for all $i \in N \cup\{0\}$. $\varphi_{k}$ is initial valued, $f$ is the differential operator and the independent variables $(x, t)$ ( where $t$ denotes time and $x$ is space variable) denote the position in space or size of cells and maturation level at a time. The solution of (1) can include the voltage, temperature, particle density, type instance, chemical substances, cells, and so on. An significant example of the model, Korteweg-devries (KdV) equation. Arising in the study of shallow water waves is as follows:

$D_{t}^{\alpha}(\psi(x, t))=b \psi \frac{\partial}{\partial x} \psi\left(a_{0} x, b_{0} t\right)+\frac{\partial^{3}}{\partial x^{3}} \psi\left(a_{1} x, b_{1} t\right), 0<\alpha<1$,

Where $b$ is constant. A further well known model, Klein-Gordon time fractional nonlinear equation with proportional delay, describes aries in quantum field theory as nonlinear wave interaction. 


$$
\begin{aligned}
D_{t}^{\alpha}(\psi(x, t))=\psi \frac{\partial^{2}}{\partial x^{2}} \psi\left(a_{0} x, b_{0} t\right)-b \psi\left(a_{1} x, b_{1} t\right) \\
-F\left(\psi\left(a_{2} x, b_{2} t\right)\right)+g(x, t), 1<\alpha<2,
\end{aligned}
$$

Where $b$ is constant, $g(x, t)$ is known analytical function, and $F$ is the nonlinear operator of $\psi(x, t)$. For details of various types of models, we refer the reader to $[25,36]$ and the references therein. To the best of my knowledge, a little literature on numerical methods used to solve the TFPDE with proportional delay, among them, Chebyshev pseudo spectral method [40], spectral collocation \& waveform relaxation methods [41], iterated pseudo spectral method [17], Differential transform method [1, 2], Variational iteration method [6] and Homotopy perturbation method [4, 25, 27, 29].

The main object of this paper is to suggest by employing STHM anlternative approximate solution of the initial valued autonomous method of TFPDE with proportional delay [25, 29, 30]. The remaining sections of this paper are arranged as follows. In section 2, we present the new integral transform and some preliminaries of fractional calculus. In section 3, we discuss the analysis of the STHM and its convergence. In section 4, applications of the STHM are presented. Finally, in section 5 some conclusions are presented.

\section{Definitions and Preliminaries}

In this section, we present the important basic definitions and properties of Shehu transformation and theory of fractional calculus.

Definition 1: [9] A real function $f(t), t>0$, is considered to be in the space $C_{\mu}, \mu \in R$ if there exists a real number $\rho>\mu$, so that $f(t)=t^{\rho} g(t)$, where $g(t) \in C([0, \infty])$.

Definition 2: [9] A function $f(t), t>0$, is said to be the space $C_{\alpha}^{m}, m \in N \cup\{0\}$, if $f^{(m)}=C_{\alpha}$.

Definition 3: $[22,35]$ The left side Riemann-Liouville fractional integral of order $\alpha$ for a function $f \in C_{\mu}, \mu \geq-1$ is defined as follows

$$
I^{\alpha} f(t)=\left\{\begin{array}{lr}
\frac{1}{\Gamma(\alpha)} \int_{0}^{t}(t-\tau)^{\alpha-1} f(\tau) d \tau, \alpha>0, t & >0, \\
f(t), & \alpha=0,
\end{array}\right.
$$

Where $\Gamma(\alpha)$ is the well-known Gamma function.

Definition 4: $[22,35]$ The Riemann-Liouville fractional derivative operator ${ }^{R} D^{\alpha}$ of order $\alpha$ for a function $f \in C_{\mu}, \mu \geq-1$ is defined as follows

$$
{ }^{R} D^{\alpha} f(t)=I^{n-\alpha} D^{n} f(t)=\frac{1}{\Gamma(n-\alpha)} \int_{0}^{t}(t-\tau)^{n-\alpha-1} f(\tau) d \tau, t>0,
$$

Where $n-1<\alpha \leq n, n \in N$.

Definition 5: [16] The fractional derivative of $f(t)$ in the Caputo sense is defined as follows

$$
\begin{aligned}
& { }^{C} D^{\alpha} f(t)=I^{n-\alpha} D^{n} f(t)=\frac{1}{\Gamma(n-\alpha)} \int_{0}^{t}(t-\tau)^{n-\alpha-1} f^{(n)}(\tau) d \tau, t>0, \\
& \quad \text { Where } n-1<\alpha \leq n, n \in N, f \in C_{-1}^{n} .
\end{aligned}
$$

Definition 6: [16] The Shehu transform of the function $f(t)$ of exponential order is defined over the set of functions:

$$
A=\left\{f(t) / \exists N, \tau_{1}, \tau_{2}>0,|f(t)|<N \exp \left(\frac{|t|}{\tau_{j}}\right), \text { if } t \in(-1)^{i} \times[0, \infty)\right\},
$$

By the following integral 
$\widehat{S}[f(t)]=F(s, u)=\int_{0}^{\infty} \exp \left(-\frac{s t}{u}\right) f(t) d t, \quad t>0$,

Some special properties of the Shehu transform are as follows:

$$
\begin{gathered}
\text { 1. } \quad \hat{S}[1]=\frac{u}{s}, \\
\text { 2. } \hat{S}\left[\frac{t^{n}}{\Gamma(n+1)}\right]=\left(\frac{u}{s}\right)^{n+1}, \quad n=0,1,2, \ldots .
\end{gathered}
$$

Definition 7: [3] The Shehu transform $\widehat{S}[f(t)]$, of the Caputo fractional derivative is given by,

$$
\widehat{S}\left[{ }^{c} D^{\alpha} f(t)\right]=\left(\frac{s}{u}\right)^{\alpha} F(s, u)-\sum_{k=0}^{n-1}\left(\frac{s}{u}\right)^{\alpha-(k+1)}\left[D^{k} f(t)\right]_{t=0}, \quad n-1<\alpha<n .
$$

\section{Shehu transform homotopy method}

In order to explain the basic idea of the technique, we consider the following general fractional partial differential equation with the initial condition of the form

$D_{t}^{\alpha} \psi(x, t)+L \psi(x, t)+N \psi(x, t)=g(x, t), t>0$,

with $m-1<\alpha \leq m$ and subject to the initial condition

$\frac{\partial^{s}}{\partial t^{s}} \psi(x, 0)=\psi^{(s)}(x, 0)=f_{s}(x), \quad s=0,1, \ldots, m-1, x \in R$.

where $D_{t}^{\alpha} \psi(x, t)$ is the Caputo fractional derivative of the function $\psi(x, t), g(x, t)$ is the source term, $L$ is the linear differential operator and $N$ is the general nonlinear differential operator. Applying the Shehu transform (denoted in this paper by $\widehat{S}$ ) on both sides of Equation (10), we get

$$
\hat{S}\left[D_{t}^{\alpha} \psi(x, t)+L \psi(x, t)+N \psi(x, t)\right]=\hat{S}[g(x, t)],
$$

and

$\hat{S}\left[D_{t}^{\alpha} \psi(x, t)\right]=\hat{S}[g(x, t)-L \psi(x, t)-N \psi(x, t)]$.

Using the Shehu transform property and the initial conditions in Equation (11), we have

$$
\left(\frac{s}{u}\right)^{\alpha}\left[\widehat{S}[\psi(x, t)]-\sum_{k=0}^{m-1}\left(\frac{s}{u}\right)^{\alpha-(k+1)} \psi^{(k)}(x, 0)\right]=\widehat{S}[g(x, t)-L \psi(x, t)-N \psi(x, t)],
$$

and

$$
\widehat{S}[\psi(x, t)]=\sum_{k=0}^{m-1}\left(\frac{s}{u}\right)^{-(k+1)} \psi^{(k)}(x, 0)+\left(\frac{u}{s}\right)^{\alpha} \hat{S}[g(x, t)-L \psi(x, t)-N \psi(x, t)],
$$

Operating with the Shehu inverse on both sides of Equation (13) gives

$$
\begin{aligned}
\psi(x, t)=\widehat{S}^{-1}\left[\sum_{k=0}^{m-1}\left(\frac{s}{u}\right)^{-(k+1)}\right. & \left.\psi^{(k)}(x, 0)+\left(\frac{u}{s}\right)^{\alpha} \hat{S}[g(x, t)]\right] \\
& -\hat{S}^{-1}\left[\left(\frac{u}{s}\right)^{\alpha} \hat{S}[L \psi(x, t)+N \psi(x, t)]\right],
\end{aligned}
$$

and

$$
\psi(x, t)=F(x, t)-\widehat{S}^{-1}\left[\left(\frac{u}{s}\right)^{\alpha} \widehat{S}[L \psi(x, t)+N \psi(x, t)]\right],
$$

where $F(x, t)$ represent the term arising from the source term and the prescribed initial conditions. Now we apply the HPM. 
$\psi(x, t)=\sum_{n=0}^{\infty} p^{n} \psi_{n}(x, t)$,

Where $p \in[0,1]$ is the homotopy parameter and the nonlinear term can be decomposed as

$N \psi(x, t)=\sum_{n=0}^{\infty} p^{n} A_{n}(\psi)$,

Where $A_{n}$ are Adomian's polynomials that are given by [38]

$A_{n}=\frac{1}{n !} \frac{d^{n}}{d p^{n}}\left[N\left(\sum_{j=0}^{\infty} p^{j} \psi_{j}(x, t)\right)\right]_{p=0}, \quad n=0,1,2, \ldots$

Substituting Equation (15) and Equation (16) in Equation (14), we get

$$
\sum_{n=0}^{\infty} p^{n} \psi_{n}(x, t)=F(x, t)-\widehat{S}^{-1}\left[p\left(\frac{u}{s}\right)^{\alpha} \widehat{S}\left[L\left(\sum_{n=0}^{\infty} p^{n} \psi_{n-1}(x, t)\right)+N\left(\sum_{n=0}^{\infty} p^{n} A_{n-1}(\psi)\right)\right]\right] \text {. }
$$

When both sides of equation (18) are compared, we get

$$
\begin{gathered}
p^{0}: \psi_{0}(x, t)=F(x, t), \\
p^{1}: \psi_{1}(x, t)=\widehat{S}^{-1}\left[\left(\frac{u}{s}\right)^{\alpha} \widehat{S}\left[-L \psi_{0}(x, t)-A_{0}(\psi)\right]\right], \\
p^{2}: \psi_{2}(x, t)=\widehat{S}^{-1}\left[\left(\frac{u}{s}\right)^{\alpha} \hat{S}\left[-L \psi_{1}(x, t)-A_{1}(\psi)\right]\right], \\
p^{n}: \psi_{n}(x, t)=\widehat{S}^{-1}\left[\left(\frac{u}{s}\right)^{\alpha} \hat{S}\left[-L \psi_{n-1}(x, t)-A_{n-1}(\psi)\right]\right] .
\end{gathered}
$$

The approximate solution for $p=1$ is given

$$
\psi(x, t)=\sum_{n=0}^{\infty} \psi_{n}(x, t) .
$$

\section{Application}

Here, we apply Shehu transform homotopy method to solve time fractional partial differential equations with proportional delay.

Example 1. Consider initial values system of time fractional order, generalized Burgers equation with proportional delay as given $[25,29,30]$

$$
\begin{aligned}
& D_{t}^{\alpha} \psi(x, t)=\frac{\partial^{2}}{\partial x^{2}} \psi(x, t)+\psi\left(\frac{x}{2}, \frac{t}{2}\right) \frac{\partial}{\partial x} \psi\left(x, \frac{t}{2}\right)+\frac{1}{2} \psi(x, t), \\
& \psi(x, 0)=x .
\end{aligned}
$$

Applying the Shehu transform on both sides of Equation (21), we get

$$
\widehat{S}\left[D_{t}^{\alpha} \psi(x, t)\right]=\widehat{S}\left[\frac{\partial^{2}}{\partial x^{2}} \psi(x, t)+\psi\left(\frac{x}{2}, \frac{t}{2}\right) \frac{\partial}{\partial x} \psi\left(x, \frac{t}{2}\right)+\frac{1}{2} \psi(x, t)\right] .
$$

Using the Shehu transform property and the initial conditions in Equation (22), we have

$$
\widehat{S}[\psi(x, t)]=x\left(\frac{u}{s}\right)+\left(\frac{u}{s}\right)^{\alpha} \widehat{S}\left[\frac{\partial^{2}}{\partial x^{2}} \psi(x, t)+\psi\left(\frac{x}{2}, \frac{t}{2}\right) \frac{\partial}{\partial x} \psi\left(x, \frac{t}{2}\right)+\frac{1}{2} \psi(x, t)\right] .
$$

Operating with the Seheu inverse on both sides of Equation (23) gives

$$
\psi(x, t)=x+\widehat{S}^{-1}\left[\left(\frac{u}{S}\right)^{\alpha} \widehat{S}\left[\frac{\partial^{2}}{\partial x^{2}} \psi(x, t)+\psi\left(\frac{x}{2}, \frac{t}{2}\right) \frac{\partial}{\partial x} \psi\left(x, \frac{t}{2}\right)+\frac{1}{2} \psi(x, t)\right]\right] .
$$


By applying the homotopy parameter and the nonlinear term can be decomposed as

$$
\sum_{n=0}^{\infty} p^{n} \psi_{n}(x, t)=x+\hat{S}^{-1}\left[p\left(\frac{u}{S}\right)^{\alpha} \hat{S}\left[\sum_{n=0}^{\infty} p^{n}\left(\psi_{n-1}\right)_{x x}+\sum_{n=0}^{\infty} p^{n} A_{n-1}(\psi)+\frac{1}{2} \sum_{n=0}^{\infty} p^{n} \psi_{n-1}\right]\right] \text {. }
$$

When both sides of equation (25) are compared, we get

$$
\begin{gathered}
p^{0}: \psi_{0}(x, t)=x, \\
p^{1}: \psi_{1}(x, t)=\widehat{S}^{-1}\left[\left(\frac{u}{s}\right)^{\alpha} \hat{S}\left[A_{0}(\psi)+\psi_{0, x x}(x, t)+\frac{1}{2} \psi_{0}(x, t)\right]\right], \\
p^{2}: \psi_{2}(x, t)=\hat{S}^{-1}\left[\left(\frac{u}{s}\right)^{\alpha} \hat{S}\left[A_{1}(\psi)+\psi_{1, x x}(x, t)+\frac{1}{2} \psi_{1}(x, t)\right]\right], \\
p^{3}: \psi_{3}(x, t)=\widehat{S}^{-1}\left[\left(\frac{u}{s}\right)^{\alpha} \widehat{S}\left[A_{2}(\psi)+\psi_{2, x x}(x, t)+\frac{1}{2} \psi_{2}(x, t)\right]\right], \\
p^{4}: \psi_{4}(x, t)=\widehat{S}^{-1}\left[\left(\frac{u}{s}\right)^{\alpha} \widehat{S}\left[A_{3}(\psi)+\psi_{3, x x}(x, t)+\frac{1}{2} \psi_{3}(x, t)\right]\right],
\end{gathered}
$$

Moreover, if we set $v=\psi\left(\frac{x}{2}, \frac{t}{2}\right), w=\psi\left(x, \frac{t}{2}\right)$ and the components of $A_{n}(\psi)$ polynomials $n=0,1,2, \ldots$, are given by

$A_{0}(\psi)=v_{0} w_{0, x}$,

$A_{1}(\psi)=v_{0} w_{1, x}+v_{1} w_{0, x}$,

$A_{2}(\psi)=v_{0} w_{2, x}+v_{1} w_{1, x}+v_{2} w_{0, x}$,

$A_{3}(\psi)=v_{0} w_{3, x}+v_{1} w_{2, x}+v_{2} w_{1, x}+v_{3} w_{0, x}$,

Using the iteration formulas (26) and (27), we obtain

$$
\begin{gathered}
p^{0}: \psi_{0}(x, t)=x, \\
p^{1}: \psi_{1}(x, t)=\frac{x t^{\alpha}}{\Gamma(\alpha+1)}, \\
p^{2}: \psi_{2}(x, t)=\frac{\left(1+2^{1-\alpha}\right) x t^{2 \alpha}}{2 \Gamma(2 \alpha+1)}, \\
p^{3}: \psi_{3}(x, t)=\left[\left(2+2^{\alpha}\right)\left(2+2^{2 \alpha}\right)+\frac{2^{\alpha+1} \Gamma(2 \alpha+1)}{\Gamma^{2}(\alpha+1)}\right] \frac{2^{-3 \alpha-2} x t^{3 \alpha}}{\Gamma(3 \alpha+1)}, \\
p^{4}: \psi_{4}(x, t)=\left[\left(2+2^{\alpha}\right)\left(2+2^{2 \alpha}\right)\left(2+2^{3 \alpha}\right)+\frac{2^{\alpha+1}\left(2+2^{3 \alpha}\right) \Gamma(2 \alpha+1)}{\Gamma^{2}(\alpha+1)}\right. \\
\left.+\frac{2^{3 \alpha+2}\left(1+2^{1-\alpha}\right) \Gamma(3 \alpha+1)}{\Gamma(\alpha+1) \Gamma(2 \alpha+1)}\right] \frac{2^{-6 \alpha-3} x t^{4 \alpha}}{\Gamma(4 \alpha+1)},
\end{gathered}
$$

Then the approximate solution of equation (21) for special case $\alpha=1$ in a closed is given by

$$
\psi(x, t)=\sum_{n=0}^{\infty} \psi_{n}(x, t)=x\left(1+t+\frac{t^{2}}{2 !}+\frac{t^{3}}{3 !}+\frac{t^{4}}{4 !}+\frac{t^{5}}{5 !}+\cdots\right) .
$$


Table 1 Comparison of fifth-order STHM solution with exact solution for Example. 1 with $\alpha=1$.

\begin{tabular}{|c|c|l|l|r|}
\hline$x$ & $t$ & Exact Sol. & STHM & \multicolumn{1}{|c|}{$E_{a b s}$} \\
\hline 0.25 & 0.25 & 0.321006354 & 0.321006266 & $8.8 E-08$ \\
\cline { 2 - 5 } & 0.5 & 0.412180317 & 0.412174479 & $5.838 E-06$ \\
\cline { 2 - 5 } & 0.75 & 0.529250004 & 0.529180908 & $6.9096 E-05$ \\
\cline { 2 - 5 } & 1 & 0.679570457 & 0.679166666 & $4.03791 E-04$ \\
\hline 0.5 & 0.25 & 0.642012708 & 0.642012532 & $1.76 E-07$ \\
\cline { 2 - 5 } & 0.5 & 0.824360635 & 0.824348958 & $1.1677 E-05$ \\
\cline { 2 - 5 } & 0.75 & 1.058500008 & 1.058361816 & $1.38192 E-04$ \\
\cline { 2 - 5 } & 1 & 1.359140914 & 1.358333333 & $8.07581 E-04$ \\
\hline 0.75 & 0.25 & 0.963019062 & 0.963018798 & $2.64 E-07$ \\
\cline { 2 - 5 } & 0.5 & 1.236540953 & 1.236523437 & $1.7516 E-05$ \\
\cline { 2 - 5 } & 0.75 & 1.587750012 & 1.587542724 & $2.07288 E-04$ \\
\cline { 2 - 5 } & 1 & 2.038711371 & 2.037500000 & $1.211371 E-03$ \\
\hline
\end{tabular}

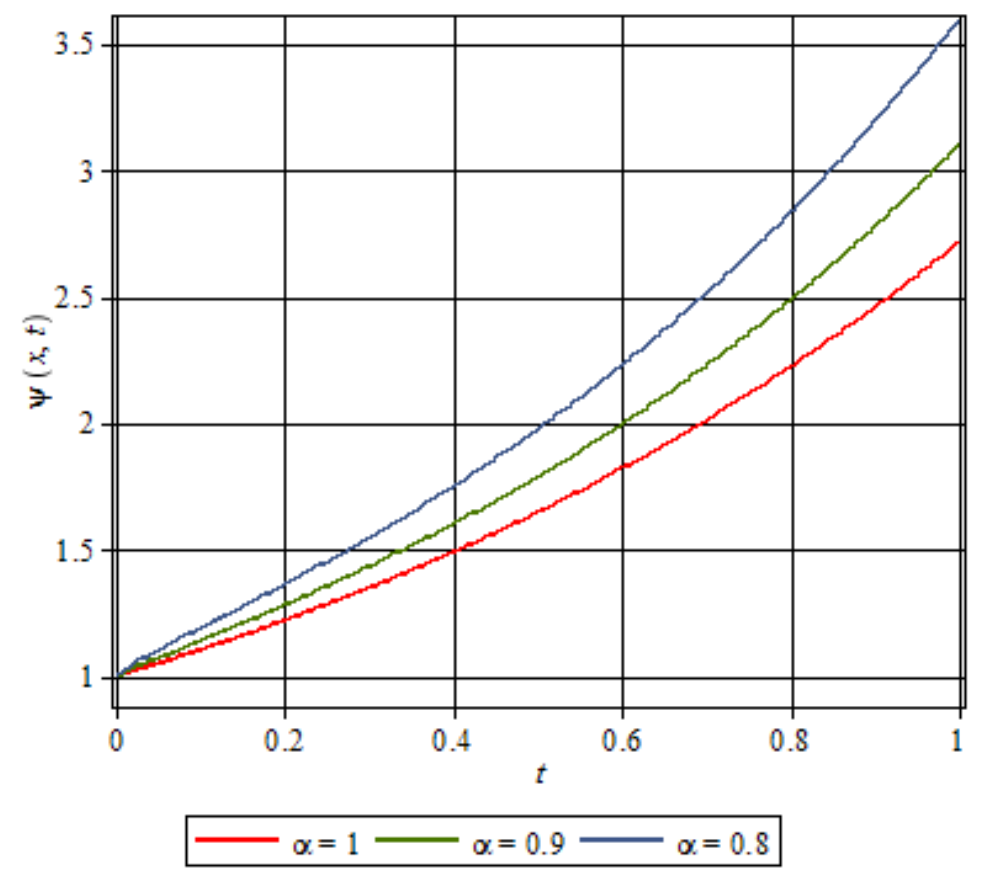

Figure 1: Plots of STHM solution $\psi(x, t)$ of Example 1 for $\alpha=0.8,0.9,1$; $\mathrm{t} \in[0,1] ; \mathrm{x}=1$. 


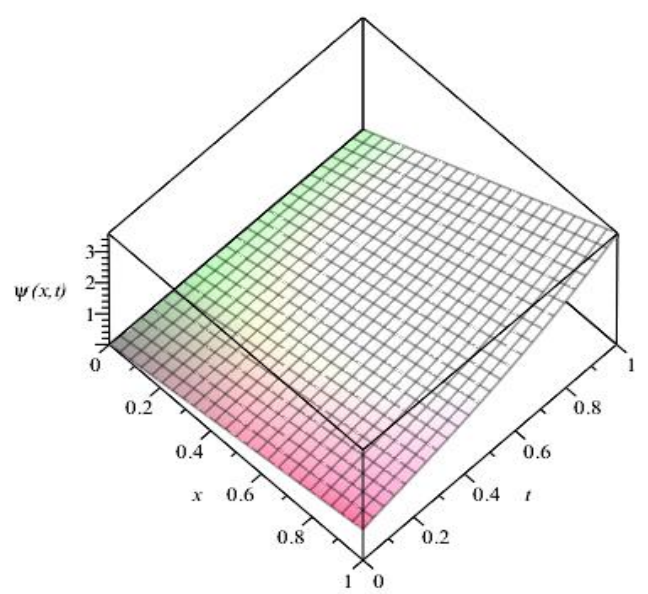

(a)

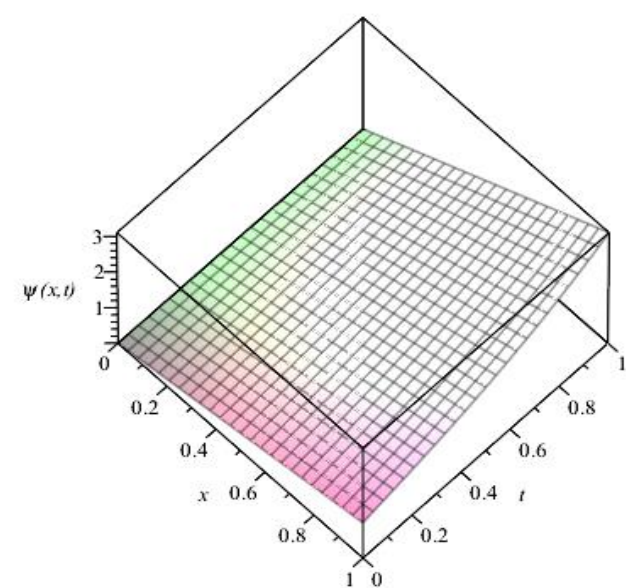

(b)

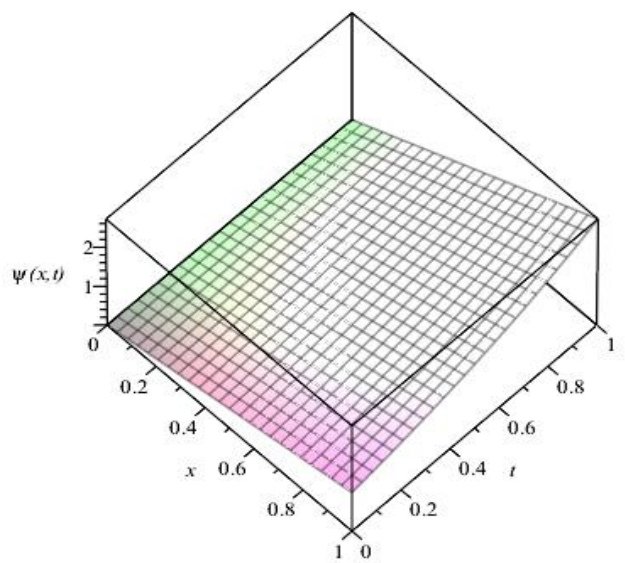

(c)

Figure 2: The behavior of the solution of STHM solution $\psi$ of Example 1 for $(a) \alpha=0.8 ;(b) \alpha=0.9 ;(c) \alpha=1$.

Hence, the exact solution of equation (21) is given by $\psi(x, t)=x e^{t}$. The exact solution is in closed agreement with the result obtained $[1,25,29,30]$.Also it is clear that form the results given in Table. 1 represent a comparison between the numerical solution of equation (21) using the STHM with the exact solution when . Following Fig. 1 represent the approximate solution of problem (21) using STHM up to five terms for different order of at different time levels with , While Figure 2 displays two dimensional plots.

Example 2. Consider initial values TFPDE with proportional delay as given [ 25, 29, 30 ]:

$$
\begin{aligned}
& D_{t}^{\alpha} \psi(x, t)=\psi\left(x, \frac{t}{2}\right) \frac{\partial^{2}}{\partial x^{2}} \psi\left(x, \frac{t}{2}\right)-\psi(x, t), \\
& \psi(x, 0)=x^{2} .
\end{aligned}
$$

Applying the Shehu transform on both sides of Equation (29), we get

$$
\widehat{S}\left[D_{t}^{\alpha} \psi(x, t)\right]=\widehat{S}\left[\psi\left(x, \frac{t}{2}\right) \frac{\partial^{2}}{\partial x^{2}} \psi\left(x, \frac{t}{2}\right)-\psi(x, t)\right] .
$$

Using the Shehu transform property and the initial conditions in Equation (30), we have

$$
\widehat{S}[\psi(x, t)]=x^{2}\left(\frac{u}{s}\right)+\left(\frac{u}{s}\right)^{\alpha} \widehat{S}\left[\psi\left(x, \frac{t}{2}\right) \frac{\partial^{2}}{\partial x^{2}} \psi\left(x, \frac{t}{2}\right)-\psi(x, t)\right] .
$$

Operating with the Shehu inverse on both sides of Equation (31) gives 


$$
\psi(x, t)=x^{2}+\hat{S}^{-1}\left[\left(\frac{u}{s}\right)^{\alpha} \hat{S}\left[\psi\left(x, \frac{t}{2}\right) \frac{\partial^{2}}{\partial x^{2}} \psi\left(x, \frac{t}{2}\right)-\psi(x, t)\right]\right] .
$$

By applying the homotopy parameter and the nonlinear term can be decomposed as

$$
\sum_{n=0}^{\infty} p^{n} \psi_{n}(x, t)=x^{2}+\widehat{S}^{-1}\left[p\left(\frac{u}{S}\right)^{\alpha} S\left[\sum_{n=0}^{\infty} p^{n} A_{n-1}(\psi)-\sum_{n=0}^{\infty} p^{n} \psi_{n-1}\right]\right] \text {. }
$$

When both sides of equation (33) are compared, we get

$$
\begin{aligned}
& p^{0}: \psi_{0}(x, t)=x^{2}, \\
& p^{1}: \psi_{1}(x, t)=\widehat{S}^{-1}\left[\left(\frac{u}{s}\right)^{\alpha} \widehat{S}\left[A_{0}(\psi)-\psi_{0}(x, t)\right]\right], \\
& p^{2}: \psi_{2}(x, t)=\widehat{S}^{-1}\left[\left(\frac{u}{s}\right)^{\alpha} \hat{S}\left[A_{1}(\psi)-\psi_{1}(x, t)\right]\right], \\
& p^{3}: \psi_{3}(x, t)=\widehat{S}^{-1}\left[\left(\frac{u}{s}\right)^{\alpha} \widehat{S}\left[A_{2}(\psi)-\psi_{2}(x, t)\right]\right], \\
& p^{4}: \psi_{4}(x, t)=\widehat{S}^{-1}\left[\left(\frac{u}{s}\right)^{\alpha} \hat{S}\left[A_{3}(\psi)-\psi_{3}(x, t)\right]\right],
\end{aligned}
$$

Moreover, if we set $v=\psi\left(x, \frac{t}{2}\right)$ and the components of $A_{n}(\psi)$ polynomials $(n=0,1,2, \ldots)$, are given by $A_{0}(\psi)=v_{0} v_{0, x x}$,

$A_{1}(\psi)=v_{0} v_{1, x x}+v_{1} v_{0, x x}$,

$A_{2}(\psi)=v_{0} v_{2, x x}+v_{1} v_{1, x x}+v_{2} v_{0, x x}$,

$A_{3}(\psi)=v_{0} v_{3, x x}+v_{1} v_{2, x x}+v_{2} v_{1, x x}+v_{3} v_{0, x x}$,

Using the iteration formulas (34) and (35), we obtain

$$
\begin{gathered}
p^{0}: \psi_{0}(x, t)=x, \\
p^{1}: \psi_{1}(x, t)=\frac{x^{2} t^{\alpha}}{\Gamma(\alpha+1)}, \\
p^{2}: \psi_{2}(x, t)=\left[2^{-\alpha}\left(2^{2}-2^{\alpha}\right)\right] \frac{x^{2} t^{2 \alpha}}{\Gamma(2 \alpha+1)}, \\
p^{3}: \psi_{3}(x, t)=\left[\left(2^{2}-2^{\alpha}\right)\left(2^{2}-2^{2 \alpha}\right)+\frac{2^{\alpha+1} \Gamma(2 \alpha+1)}{\Gamma^{2}(\alpha+1)}\right] \frac{2^{-3 \alpha} x^{2} t^{3 \alpha}}{\Gamma(3 \alpha+1)}, \\
p^{4}: \psi_{4}(x, t)=\left[\left(2^{2}-2^{\alpha}\right)\left(2^{2}-2^{2 \alpha}\right)\left(2^{2}-2^{3 \alpha}\right)+\frac{2^{\alpha+1}\left(2^{2}-2^{3 \alpha}\right) \Gamma(2 \alpha+1)}{\Gamma^{2}(\alpha+1)}\right. \\
\left.+\frac{2^{2 \alpha+2}\left(2^{2}-2^{\alpha}\right) \Gamma(3 \alpha+1)}{\Gamma(\alpha+1) \Gamma(2 \alpha+1)}\right] \frac{2^{-6 \alpha} x^{2} t^{4 \alpha}}{\Gamma(4 \alpha+1)},
\end{gathered}
$$

Then the approximate solution of equation (29) for special case $\alpha=1$ in a closed is given by

$$
\psi(x, t)=\sum_{n=0}^{\infty} \psi_{n}(x, t)=x^{2}\left(1+t+\frac{t^{2}}{2 !}+\frac{t^{3}}{3 !}+\frac{t^{4}}{4 !}+\frac{t^{5}}{5 !}+\cdots\right) .
$$


Table 2 Comparison of fifth-order STHM solution with exact solution for Example. 2 with $\alpha=1$.

\begin{tabular}{|c|c|l|l|r|}
\hline$x$ & $t$ & Exact Sol. & STHM & \multicolumn{1}{|c|}{$E_{\text {abs }}$} \\
\hline 0.25 & 0.25 & 0.080251588 & 0.080251566 & $2.2 E-08$ \\
\cline { 2 - 5 } & 0.5 & 0.103045079 & 0.103043619 & $1.459 E-06$ \\
\cline { 2 - 5 } & 0.75 & 0.132312501 & 0.132295227 & $1.7274 E-05$ \\
\cline { 2 - 5 } & 1 & 0.169892614 & 0.169791666 & $1.00948 E-04$ \\
\hline 0.5 & 0.25 & 0.321006354 & 0.321006266 & $8.8 E-08$ \\
\cline { 2 - 5 } & 0.5 & 0.412180317 & 0.412174479 & $5.838 E-06$ \\
\cline { 2 - 5 } & 0.75 & 0.529250004 & 0.529180908 & $6.9096 E-05$ \\
\cline { 2 - 5 } & 1 & 0.679570457 & 0.679166666 & $4.03790 E-04$ \\
\hline 0.75 & 0.25 & 0.722264296 & 0.722264099 & $1.97 E-07$ \\
\cline { 2 - 5 } & 0.5 & 0.927405714 & 0.927392578 & $1.3136 E-05$ \\
\cline { 2 - 5 } & 0.75 & 1.190812509 & 1.190657043 & $1.55466 E-04$ \\
\cline { 2 - 5 } & 1 & 1.529033528 & 1.528125000 & $9.08528 E-04$ \\
\hline
\end{tabular}

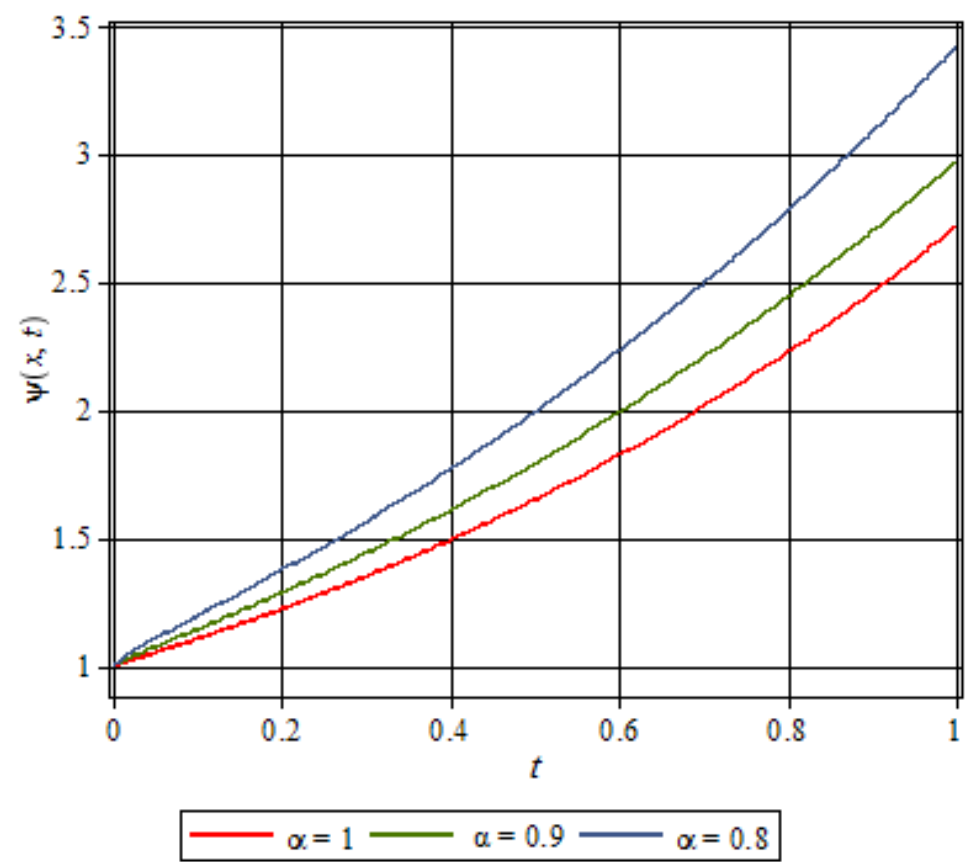

Figure 3: Plots of STHM solution $\psi(x, t)$ of Example 2 for $\alpha=0.8,0.9,1$; $\mathrm{t} \in[0,1] ; \mathrm{x}=1$. 


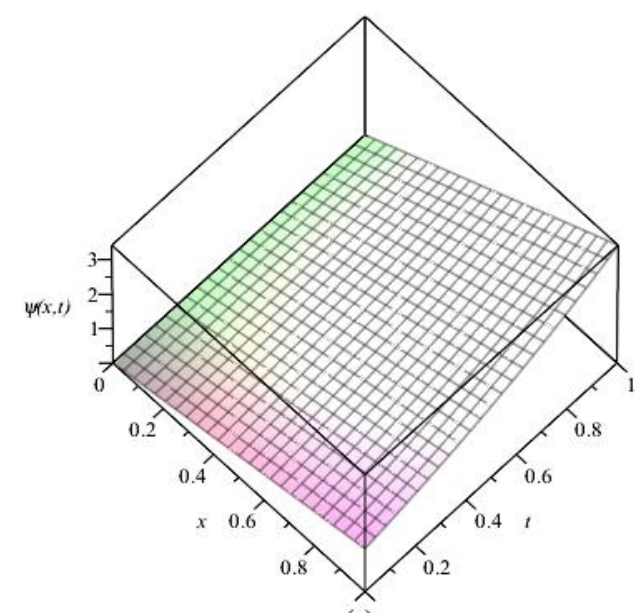

(a)

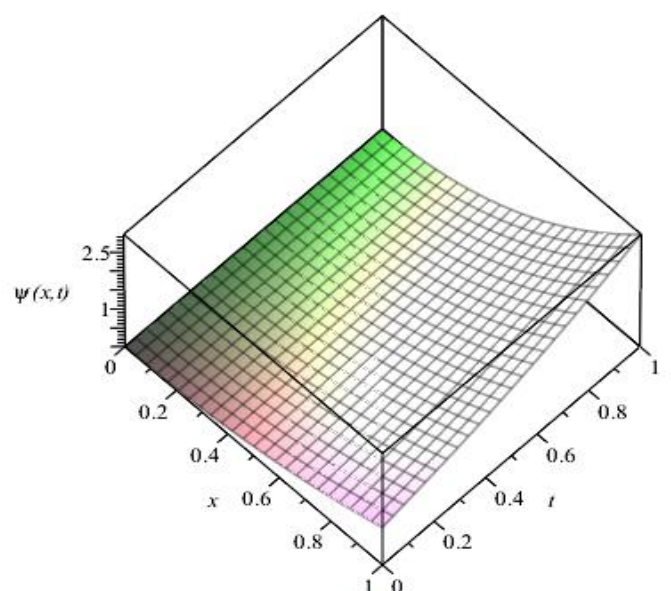

(b)

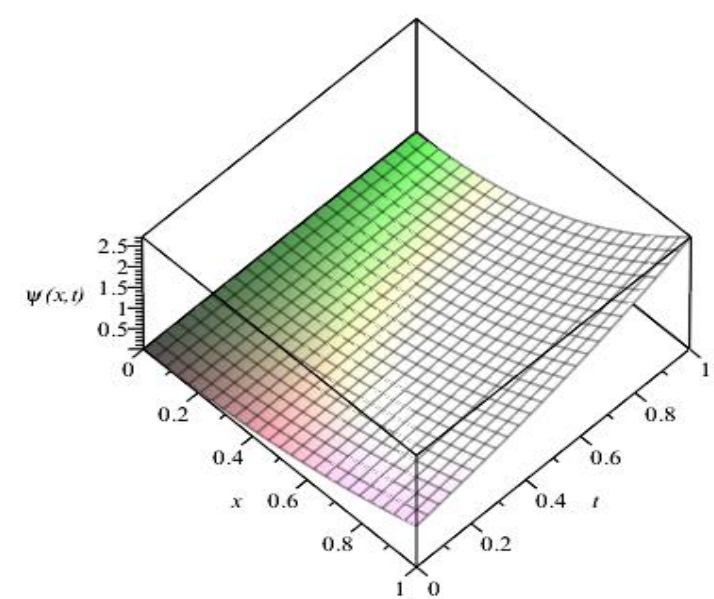

(c)

Figure 4: The behavior of the solution of STHM solution u of Example 2 for $(a) \alpha=0.8 ;(b) \alpha=0.9 ;(c) \alpha=1$.

Hence, the exact solution of equation (29) is given by $\psi(x, t)=x^{2} e^{t}$. The exact solution is in closed agreement with the result obtained $[1,25,29,30]$. Also it is clear that form the results given in Table.2 represent a comparison between the numerical solution of equation (29) using the STHM with the exact solution when . Following Fig. 3 represent the approximate solution of problem (29) using STHM up to five terms for different order of at different time levels with , While Figure 4 displays two dimensional plots.

Example 3. Consider initial values TFPDE with proportional delay as given $[25,29,30]$

$$
\begin{aligned}
& D_{t}^{\alpha} \psi(x, t)=\frac{\partial^{2}}{\partial x^{2}} \psi\left(\frac{x}{2}, \frac{t}{2}\right) \frac{\partial}{\partial x} \psi\left(\frac{x}{2}, \frac{t}{2}\right)-\frac{1}{8} \frac{\partial}{\partial x} \psi(x, t)-\psi(x, t), \\
& \psi(x, 0)=x^{2} .
\end{aligned}
$$

Applying the Shehu transform on both sides of Equation (37), we get

$\widehat{S}\left[D_{t}^{\alpha} \psi(x, t)\right]=\widehat{S}\left[\frac{\partial^{2}}{\partial x^{2}} \psi\left(\frac{x}{2}, \frac{t}{2}\right) \frac{\partial}{\partial x} \psi\left(\frac{x}{2}, \frac{t}{2}\right)-\frac{1}{8} \frac{\partial}{\partial x} \psi(x, t)-\psi(x, t)\right]$.

Using the Shehu transform property and the initial conditions in Equation (38), we have

$$
\widehat{S}[\psi(x, t)]=x^{2}\left(\frac{u}{s}\right)+\left(\frac{u}{s}\right)^{\alpha} \hat{S}\left[\frac{\partial^{2}}{\partial x^{2}} \psi\left(\frac{x}{2}, \frac{t}{2}\right) \frac{\partial}{\partial x} \psi\left(\frac{x}{2}, \frac{t}{2}\right)-\frac{1}{8} \frac{\partial}{\partial x} \psi(x, t)-\psi(x, t)\right] .
$$

Operating with the Seheu inverse on both sides of Equation (39) gives 


$$
\psi(x, t)=x^{2}+\widehat{S}^{-1}\left[\left(\frac{u}{s}\right)^{\alpha} \hat{S}\left[\frac{\partial^{2}}{\partial x^{2}} \psi\left(\frac{x}{2}, \frac{t}{2}\right) \frac{\partial}{\partial x} \psi\left(\frac{x}{2}, \frac{t}{2}\right)-\frac{1}{8} \frac{\partial}{\partial x} \psi(x, t)-\psi(x, t)\right]\right] .
$$

By applying the homotopy parameter and the nonlinear term can be decomposed as

$$
\sum_{n=0}^{\infty} p^{n} \psi_{n}(x, t)=x^{2}+\widehat{S}^{-1}\left[p\left(\frac{u}{s}\right)^{\alpha} \hat{S}\left[\sum_{n=0}^{\infty} p^{n} A_{n-1}(\psi)-\frac{1}{8} \sum_{n=0}^{\infty} p^{n}\left(\psi_{n-1}\right)_{x}-\sum_{n=0}^{\infty} p^{n}\left(\psi_{n-1}\right)\right]\right] \text {. }
$$

When both sides of equation (41) are compared, we get

$$
\begin{aligned}
& p^{0}: \psi_{0}(x, t)=x^{2}, \\
& p^{1}: \psi_{1}(x, t)=\widehat{S}^{-1}\left[\left(\frac{u}{s}\right)^{\alpha} \hat{S}\left[A_{0}(\psi)-\frac{1}{8} \psi_{0, x}(x, t)-\psi_{0}(x, t)\right]\right], \\
& p^{2}: \psi_{2}(x, t)=\widehat{S}^{-1}\left[\left(\frac{u}{s}\right)^{\alpha} \hat{S}\left[A_{1}(\psi)-\frac{1}{8} \psi_{1, x}(x, t)-\psi_{1}(x, t)\right]\right], \\
& p^{3}: \psi_{3}(x, t)=\widehat{S}^{-1}\left[\left(\frac{u}{s}\right)^{\alpha} \hat{S}\left[A_{2}(\psi)-\frac{1}{8} \psi_{2, x}(x, t)-\psi_{2}(x, t)\right]\right], \\
& p^{4}: \psi_{4}(x, t)=\widehat{S}^{-1}\left[\left(\frac{u}{s}\right)^{\alpha} \hat{S}\left[A_{3}(\psi)-\frac{1}{8} \psi_{3, x}(x, t)-\psi_{3}(x, t)\right]\right],
\end{aligned}
$$

Moreover, if we set $v=\psi\left(\frac{x}{2}, \frac{t}{2}\right)$ and the components of $A_{n}(\psi)$ polynomials $(n=0,1,2, \ldots)$, are given by $A_{0}(\psi)=v_{0, x x} v_{0, x}$,

$A_{1}(\psi)=v_{0, x x} v_{1, x}+v_{1, x x} v_{0, x}$,

$A_{2}(\psi)=v_{0, x x} v_{2, x}+v_{1, x x} v_{1, x}+v_{2, x x} v_{0, x}$,

Using the iteration formulas (42) and (43), we obtain

$$
\begin{aligned}
& p^{0}: \psi_{0}(x, t)=x, \\
& p^{1}: \psi_{1}(x, t)=-\frac{x^{2} t^{\alpha}}{\Gamma(\alpha+1)}, \\
& p^{2}: \psi_{2}(x, t)=\frac{\left(1-2^{1-\alpha}+4 x\right) x t^{2 \alpha}}{4 \Gamma(2 \alpha+1)}, \\
& p^{3}: \psi_{3}(x, t)=\left[\left(2^{\alpha}-2\right)\left(2-2^{2 \alpha}\right)+2^{\alpha+4}\left(1+2^{\alpha}-2^{2 \alpha}+\frac{\Gamma(2 \alpha+1)}{2 \Gamma^{2}(\alpha+1)}\right) x-2^{3 \alpha+5} x^{2}\right] \frac{2^{-3 \alpha-5} t^{3 \alpha}}{\Gamma(3 \alpha+1)},
\end{aligned}
$$

Then the approximate solution of equation (21) for special case $\alpha=1$ in a closed is given by

$$
\psi(x, t)=\sum_{n=0}^{\infty} \psi_{n}(x, t)=x\left(1+t+\frac{t^{2}}{2 !}+\frac{t^{3}}{3 !}+\frac{t^{4}}{4 !}+\frac{t^{5}}{5 !}+\cdots\right) .
$$

Table 3 Comparison of fifth-order STHM solution with exact solution for Example. 3 with $\alpha=1$.

\begin{tabular}{|c|c|l|l|c|}
\hline$x$ & $t$ & Exact Sol. & STHM & $E_{a b s}$ \\
\hline 0.25 & 0.25 & 0.048675048 & 0.048675029 & $1.9 E-08$ \\
\cline { 2 - 5 } & 0.5 & 0.037908166 & 0.037906901 & $1.265 E-06$ \\
\cline { 2 - 5 } & 0.75 & 0.029522909 & 0.029508972 & $1.3937 E-05$ \\
\cline { 2 - 5 } & 1 & 0.022992465 & 0.022916667 & $7.5798 E-05$ \\
\hline
\end{tabular}




\begin{tabular}{|c|c|c|c|r|}
\hline 0.5 & 0.25 & 0.194700195 & 0.194700114 & $8.1 E-08$ \\
\cline { 2 - 5 } & 0.5 & 0.151632664 & 0.151627604 & $5.06 E-06$ \\
\cline { 2 - 5 } & 0.75 & 0.118091638 & 0.118035889 & $5.5749 E-05$ \\
\cline { 2 - 5 } & 1 & 0.0919698602 & 0.091666667 & $3.031932 E-04$ \\
\hline 0.75 & 0.25 & 0.438075440 & 0.438075256 & $1.84 E-07$ \\
\cline { 2 - 5 } & 0.5 & 0.341173496 & 0.341162109 & $1.1387 E-05$ \\
\cline { 2 - 5 } & 0.75 & 0.265706185 & 0.265580749 & $1.25436 E-04$ \\
\cline { 2 - 5 } & 1 & 0.206932185 & 0.206250000 & $6.82185 E-04$ \\
\hline
\end{tabular}

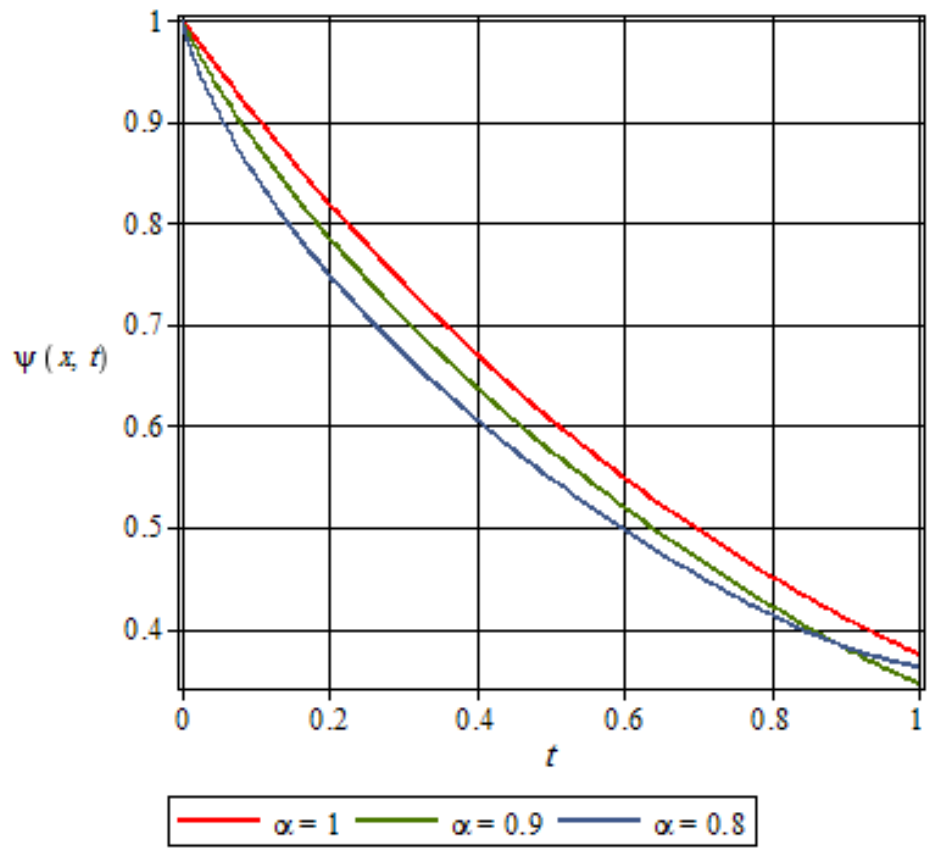

Figure 5: Plots of STHM solution $\psi(x, t)$ of Example 3 for $\alpha=0.8,0.9$, $1 ; \mathrm{t} \in[0,1] ; \mathrm{x}=1$. 


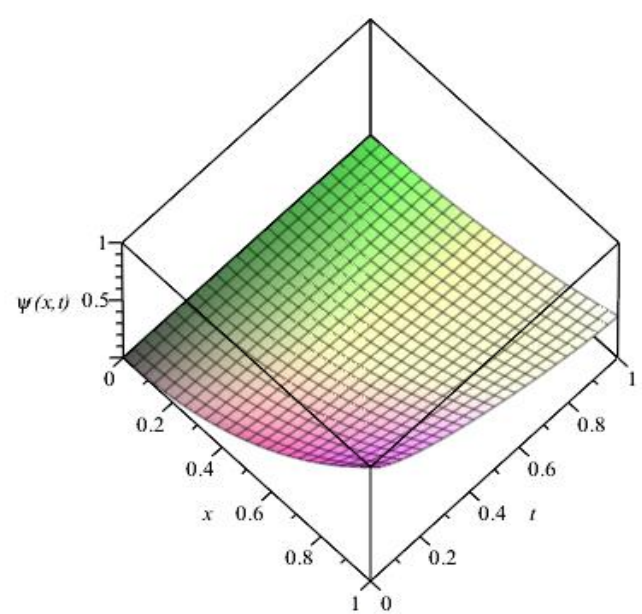

(a)

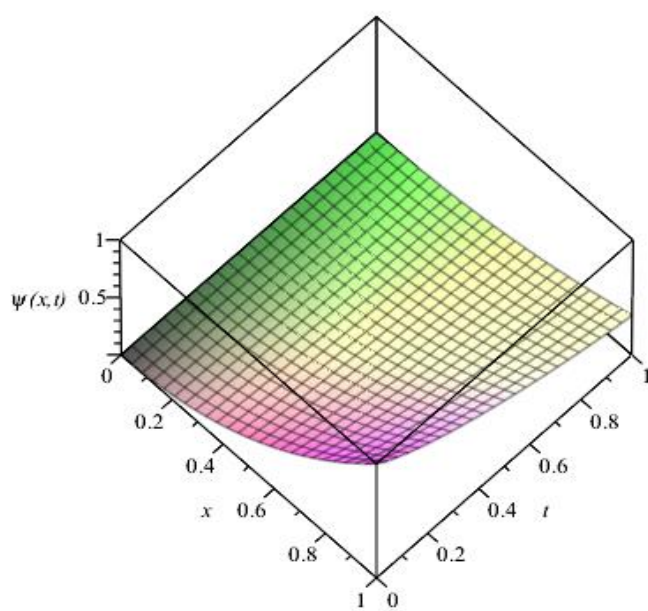

(b)

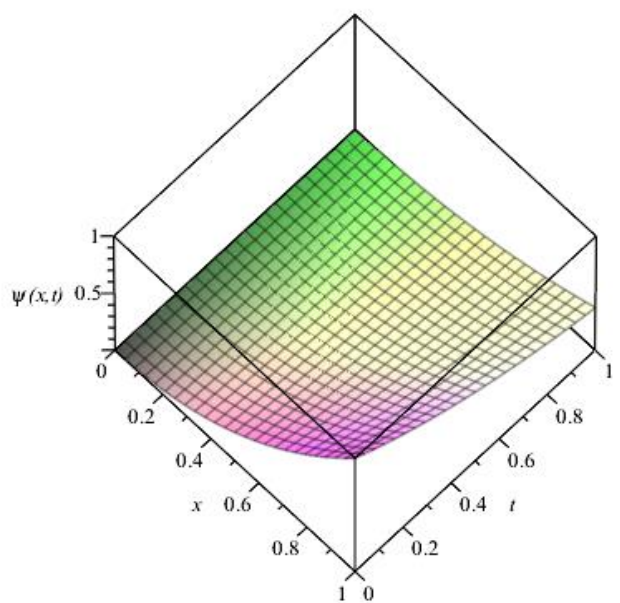

(c)

Figure 6: The behavior of the solution of STHM solution $\psi$ of Example 3 for $(a) \alpha=0.8 ;(b) \alpha=0.9 ;(c) \alpha=1$.

Hence, the exact solution of equation (37) is given by $\psi(x, t)=x^{2} e^{-t}$. The exact solution is in closed agreement with the result obtained $[1,25,29,30]$. Also it is clear that form the results given in Table.3 represent a comparison between the numerical solution of equation (37) using the STHM with the exact solution when . Following Fig. 5 represent the approximate solution of problem (37) using STHM up to five terms for different order of at different time levels with , While Figure 6 displays two dimensional plots.

\section{Conclusion}

Shehu transform homotopy method is successfully used in this paper for numerical computation of the initial valued autonomous time fractional TFPDEs model system with proportional delay, where we use the fractional derivative in Caputo sense. There are three test problems carried to validate and illustrate the methods efficiency. The solutions proposed obtained excellent agreement with $[1,25,29,30]$. These approximate solutions are obtained without any discretization, perturbation restrictive conditions, which converge very quckly.

\section{Conflict of Interest}

The authors have not declared conflict of interest.

\section{ACKNOWLEDGEMENT}

The authors wish to thank referees for their comments. 


\section{References}

1. R. Abazari, M. Ganji, Extended two-dimensional DTM and its application on nonlinear PDEs with proportional delay, Int. J. Comput. Math. 88 (8) (2011): 1749-1762. DOI: 10.1080/00207160.2010.526704.

2. R. Abazari, A. Kilicman, Application of differential transform method on nonlinear integro-differential equations with proportional delay, Neural Comput \& Applic. 24 (2014): 391-397. http://dx.doi.org/10.1007/s00521-012-1235-4.

3. R. Belgacem, D. Baleanu and Bokhari, Shehu transform and applications to Caputo fractional differential equations, International journal of Analysis and Applications. 17 (2019): 917-927. http://doi.org/10.28924/2292-8639-17-2019-917.

4. J. Biazar, B. Ghanbari, The homotopy perturbation method for solving neutral functional-differential equations with proportional delays, J. King Saud Univ. Sci. 24: (2010) 33-37. http://doi.org/10.1016/j.jksus.2010.07.026.

5. M. Caputo, F. Mainardi, Linear models of dissipation in anelastic solids. Rivista del Nuovo Cimento. 1: (1971) 161-98.

6. X. Chen, L. Wang, The variational iteration method for solving a neutral functional-differential equation with proportional delays, Comput. Math. Appl. 59: (2010) 2696-2702. http://doi.org/10.1016/j.jcamwa.2010.01.037.

7. J. H. He, Nonlinear oscillation with fractional derivative and its applications, Int. Conf. Vibrating Engg'98, Dalian, (1998) 288-91.

8. J. H. He, Homotopy perturbation technique, Comput. Methods Appl. Mechanics Engg. 178: (1999) 25762.

9. H. Jafari, M. Nazari, D. Baleanu and C. M. Khalique, A new approach for solving a system of fractional partial differential equation, Computer and Mathematics with Application. 66 (2012), 838-843. [1] http://doi.org/10.1016/j.jcamwa.2012.11.014.

10. Andre A. Keller, Contribution of the delay differential equations to the complex economic macrodynamics, WSEAS Transactions on Systems. 9(4): (2010) 258-271.

11. A. Khalouta and A. Kadem, A new method to solve fractional differential equations: Inverse fractional Shehu transform method, Applications and Applied Mathematics. 14(2): (2019) 926-941.

12. A. A. Kilbas, H. M. Srivastava, J. J. Trujillo, Theory and applications of fractional differential equations. Elsevier, Amsterdam 9(2006).

13. J. Liu, G. Hou, Numerical solutions of the space-and time-fractional coupled burgers equations by generalized differential transform method, Appl. Math. Comput. 217 (16) (2011): 7001-7008 .DOI: 10.1016/j.amc.2011.01.111.

14. A. M. S. Mahdy, A. S. Mohamed, A. A. H. Mtawa, Implementation of the Homotopy Perturbation Sumudu Transform Method for Solving Klein-Gordon Equation, Applied Mathematics. 6 (2015): 617628. http://dx.doi.org/10.4236/am.2015.63056.

15. [A. M. S. Mahdy, A. S. Mohamed, A. A. H. Mtawa, Variational homotopy perturbation method for solving the generalized time-space fractional Schrödinger equation, International journal of Physical sciences. 10(11) (2015): 342-350. https://doi.org/10.5897/IJPS2015.4287.

16. S. Maitama and W. Zhao, New integral transform: Shehu transform a generalization of Sumudu and Laplace transform for solving differential equations, International Journal of Analysis and Applications, 17(2) (2019): 167-190. DOI: 10.10.28924/2291-8639-17-2019-167.

17. J. Mead, B. Zubik-Kowal, An iterated pseudospectral method for delay partial differential equations, Appl. Numer. Math. 55 (2005): 227-250. http://doi.org/10.1016/j.apnum.2005.02.010. 
18. K. S. Miller and B. Ross, An Introduction to the fractional calculus and fractional differential equations, Wiley, New York. (1993).

19. [S. Momani, G.H. Erjaee, M.H. Alnasr, The modified homotopy perturbation method for solving strongly nonlinear oscillators, Computers and Mathematics with Applications. 58 (2009): 22092220.http://doi.org/10.1016/j.camwa.2009.03.082.

20. S. Momani, Z. Odibat, Analytical solution of a time-fractional Navier-Stokes equation by adomaian decomposition method, Appl. Math. Comput. 177 (2005): 488 - 494 http://doi.org/10.1016/j.amc.2005.11.025.

21. A. A. H. Mtawal, S. E. Muhammed and A. A. Almabrok, Application of the alternative variational iteration method to solve delay differential equations, International journal of Physical sciences. 15(3) (2020): 112-119. https://doi.org/10.5897/IJPS2020.4879.

22. I. Podlubny, Fractional differential equations, Academic Press, San Diego (1999).

23. M. G. Sakar, F. Erdogan, The homotopy analysis method for solving the time-fractional FornbergWhitham equation and comparison with Adomian's decomposition method, Appl. Math. Model. 37 (2021) (2013): 1634-1641. http://doi.org/10.1016/j.apm.2013.03.074.

24. M. G. Sakar, H. Erg"oren, Alternative variational iteration method for solving the time-fractional Fornberg- Whitham equation, Appl. Math. Model. 39 (14) (2015): 3972-3979. DOI: $10.1016 /$ j.apm.2014.11.048

25. M. G. Sakar, F. Uludag, F. Erdogan. (2016). Numerical solution of time-fractional nonlinear PDEs with proportional delays by homotopy perturbation method. Appl. Math. Modelling. 40 (2016): 6639-6649. http://dx.doi.org/10.1016/j.apm.2016.02.005.

26. A. Saravanan, N. Magesh, An efficient computational technique for solving the Fokker-Planck equation with space and time fractional derivative,. Journal of King Saud University Science. 28 (2015): 160 166.http://dx.doi.org/10.1016/j.jksus.2015.01.003.

27. F. Shakeri, M. Dehghan, Solution of delay differential equations via a homotopy perturbation method, $\begin{array}{lllll}\text { Mathematical and } & \text { Computer } & \text { 486-498. }\end{array}$ http://dx.doi.org/10.1016/j.mcm.2007.09.0016.

28. [B. K. Singh and P. Kumar, Homotopy perturbation transform method for fractional gas dynamics equation using Sumudu transform, Abstract and Applied Analysis. Vol. 2013, pages 8. https://doi.org/10.1155/2013/934060.

29. B. K. Singh and P. Kumar. Homotopy perturbation transform method for solving fractional partial differential equations with proportional delays, International Journal of Differential Equations. Vol. 2017, pages 11. https://arxiv.org/abs/1611.06488vl.

30. B. K. Singh and P. Kumar. (2017). Fractional variational iteration method for solving fractional partial differential equations with proportional delays, https://doi.org/10.1155/2017/5206380.

31. B. K. Singh, V. K. Srivastava, Approximate series solution of multi dimensional, time fractional-order (heatlike) diffusion equations using FRDTM, R. Soc. Open Sci. (2015). http://dx.doi.org/10.1098/rsos.140511.

32. V.K. Srivastava, S. Kumar, M.K. Awasthi, B.K. Singh, Two dimensional time fractional-order biological population model and its analytical solution, Egyptian Journal of Basic and Applied Sciences 1 (2014): 71-76.http://dx.doi.org/10.1016/j.eibas.2014.03.001.

33. V. K. Srivastava, N. Mishra, S. Kumar, B. K. Singh, M. K. Awasthi, Reduced differential transform method for solving $(1+n)$-Dimensional Burgers' equation, Egyptian Journal of Basic and Applied Sciences 1 (2014): 115-119. http://dx.doi.org/10.1016/j.eibas.2014.05.001. 
34. T. G. Thange and A. R. Gade. (2020). A new approach for solving a system of fractional partial differential equation, Advances in Mathematics. 9(4) (2020): 1933-1944. https://doi.org/10.37418/amsj.9.4.52.

35. T. G. Thange and A. R. Gade, On the Aoodh transform of fractional differential operator, Malaya Journal of Mathematik, 8(1) (2020): 225-229.

36. J. Wu, Theory and Applications of Partial Functional Differential Equations, Springer-Verlag, New York (1996).

37. X.J. Yang, D. Baleanu, Y. Khan, S.T. Mohyud-din, Local fractional variational iteration method for diffusion and wave equations on cantor sets, Rom. J. Phys. 59 (1-2) (2014): 36-48.

38. Y. Zhu, Q. Chang, S. Wu, A new algorithm for calculating Adomian polynomials, Applied Mathematics and Computation, 169 (1) (2005): 402-416. DOI: 10.1016/j.amc.2004.09.082.

39. [39] D. Ziane, R. Belgacem and A. Bokhari, A new modified Adomian decomposition method for nonlinear partial differential equations. Open J. Math. Anal. 3(2) (2019): 81-90. DOI: 10.30538/psrpoma2019.0041.

40. B. Zubik-Kowal, Chebyshev pseudo spectral method and waveform relaxation for differential and differential functional parabolic equations, Appl. Numer. Math. 34 (2-3) (2000): 309328.DOI: 10.1016/S0168-9274(99)00135-X

41. [41] B. Zubik-Kowal, Z. Jackiewicz, Spectral collocation and waveform relaxation methods for nonlinear delay partial differential equations, Appl. Numer. Math. 56 (3-4 (2005)): 433-443. http://dx.doi.org/10.1016/j.apnum.2005.04.0021. 